\title{
Un modelo explicativo de la conducta hacia la enfermedad mental
}

\author{
Sarah García-Sílberman, D ra en Psic..$^{(1)}$
}

\section{García-Sílberman S. \\ Un modelo explicativo de la conducta hacia la enfermedad mental. \\ Salud Publica Mex 2002;44:289-296.}

El texto completo en inglés de este artículo está disponible en: http://www.insp.mx/salud/index.html

\begin{abstract}
Resumen
Objetivo. Probar un modelo teórico diseñado para explicar las actitudes en relación con la enfermedad mental, a partir del conocimiento de las principales variables relacionadas con dicho constructo. Material y métodos En 1996 se efectuó una encuesta estratificada por nivel socioeconómico, edad y género en una muestra de 800 sujetos de la población general de la Ciudad de México,en relación con las creencias, actitudes e intenciones conductuales respecto de los trastornos mentales. Se construyó y validó un instrumento de medición, específico para el estudio, integrado por 120 reactivos tipo Likert, con cinco opciones de respuesta. Los datos se codificaron y analizaron estadísticamente con el paquete SPSS. La consistencia interna de las escalas utilizadas se midió por medio del Alpha de Cronbach y la validez del constructo, mediante análisis factoriales. Se efectuaron pruebas de t de student y anova, para comparar los grupos de los diferentes estratos de la muestra. Resultados Los datos obtenidos confirman la capacidad predictiva del modelo en cuanto a la cadena causal que conecta creencias, actitudes e intenciones conductuales; sin embargo, las demás variables investigadas no contribuyeron a explicarlo y la conducta resultó poco influenciada por las intenciones, dependiendo más bien de la necesidad experimentada. Conclusiones. Los resultados constituyen una base para comprender las actitudes de temor y vergüenza
\end{abstract}

\author{
García-Sílberman S. \\ An explanatory model of behavior \\ towards mental illness. \\ Salud Publica Mex 2002;44:289-296. \\ The English version of this paper \\ is available at: http://www.insp.mx/salud/index.html
}

\begin{abstract}
A bst ract
Objective. To evaluate a theoretical model designed to explain behaviors toward mental illness, considering some variables related to the construct. Material and Methods. A survey was conducted in 1996 on mental disorder beliefs, attitudes, and behavioral intentions. The sample population was stratified by so cioeconomic status, age, and gender. Study subjects were 800 individuals from Mexico City's general population. A data collection instrument was constructed and validated, consisting of 120 Likert-type items with five options each. Data were coded and analyzed with the software package SPSS. Internal consistency of the scales was assessed using Cronbach's alpha and construct validity with factorial analysis. Student's t test and AN OVA were used to compare the groups in the different strata. Results. The model allowed to confirm the predictive capacity of the causal chain connecting beliefs, attitudes, and intentions; nevertheless, other study variables did not contribute to explain it, and behavior was scarcely influenced by intentions, depending mainly on experimented necessity. Conclusions Study findings constitute a basis to understand the attitudes of shame and fear usually related to mental illnesses, to plan efficient actions aimed at modifying them, and to design programs to promote mental health.The English version of this paper is available at: http://www.insp.mx/ salud/index.html
\end{abstract}

Trabajo presentado en el IX Congreso Nacional de Investigación en Salud Pública, celebrado del 5 al 7 de marzo de 2001.

El desarrollo de este proyecto fue posible gracias al apoyo financiero del Consejo N acional de Ciencia y Tecnología, clave 2072-H9302, y el Instituto N acional de Psiquiatría Ramón de la Fuente, clave 1492.

(1) Instituto N acional de Psiquiatría Ramón de la Fuente, México, D.F., México.

Fecha de recibido: 26 de abril de 2001 • Fecha de aprobado: 14 de diciembre de 2001

Solicitud de sobretiros: D ra Sarah García-Sílberman. Jefa del Centro de Información en Salud Mental y Adicciones, Dirección de Investigaciones Epidemiológicas y Psicosociales, Instituto N acional de Psiquiatría Ramón de la Fuente, Calzada México-Xochimilco N 0. 101, Colonia San Lorenzo Huipulco,Tlalpan, 14370 México, D.F., México.

Correo electrónico: silberg@imp.edu.mx 
en relación con las enfermedades mentales, planear acciones eficaces para su modificación y diseñar programas de promoción de la salud mental. El texto completo en inglés de este artículo está disponible en: http://www.insp.mx/salud/ index.html

Palabras clave: salud mental; enfermedad mental; actitudes; conducta; modelo teórico; México
Key words: mental health; mental illness; attitudes; behavio ur; theoretical model; Mexico
$\mathrm{E}$ n el marco de la tendencia actual a la reinserción social de los enfermos mentales, aumenta la presión para que su cuidado recaiga en la familia y la comunidad..$^{1-4}$ El éxito de dicha estrategia depende en gran medida de las actitudes de la población al respecto. Bajo esta premisa, en los últimos 50 años se han desarrollado numerosos estudios para conocer las actitudes de diferentes grupos sociales en relación con las enfermedades mentales. La revisión de la literatura especializada nos muestra cientos de estudios provenientes de las más diversas culturas, incluidos algunos realizados en nuestro país. ${ }^{5-18}$ Diversas revisiones comprehensivas reseñan sus resultados. ${ }^{19-23}$

Sin embargo, a pesar de que la investigación muestra a lo largo del tiempo una consistente modificación de las actitudes en un sentido positivo, predominando en la actualidad manifestaciones de aceptación y comprensión de las enfermedades mentales, la conducta de la población no parece corresponder con las actitudes expresadas. En busca de una explicación para dicha inconsistencia, se desarrolló una investigación con el propósito de diseñar un modelo teórico que permitiera analizar las relaciones entre las diversas variables relacionadas con las actitudes, y la conducta en relación con la enfermedad mental.

En la amplia literatura publicada sobre el tema, se aprecia un predominio de investigaciones cuantitativas, descriptivas y correlacionales en términos de variables sociodemográficas; ${ }^{24-37}$ sin embargo, la mayor parte de ellas no aclara cuál es el marco teórico en que se fundamenta en relación con el constructo "actitud", su formación, estructura y modificación. Por otra parte, ningún estudio reportado incluye una aproximación a la relación entre las creencias, las actitudes y las intenciones conductuales, así como a cualesquiera otras variables intervinientes en esta relación.

Para cubrir tales carencias, esto es, la de estudios descriptivos recientes en el ámbito nacional y propuestas explicativas en el contexto internacional, se consideró pertinente plantear un marco teórico apropiado, que permitiera el diseño de un esquema o modelo teórico $^{38}$, para explicar mejor las conductas observadas en relación con la enfermedad mental.
Se consideró como base un marco conceptual, desarrollado por Fishbein y Ajzen, ${ }^{39-41}$ que plantea que las actitudes están integradas por tres componentes, uno cognitivo, uno afectivo y uno conativo, que corresponden a las creencias, actitudes e intenciones conductuales, respectivamente. Bajo este marco se propone la existencia de una cadena causal en la que el comportamiento está determinado por las intenciones de desarrollar una conducta específica; éstas dependen de las actitudes, y, a su vez, son función de las creencias sobresalientes. La premisa básica es que se tienen ciertas creencias acerca de la enfermedad mental, que determinan las actitudes, o sea, la predisposición a reaccionar en determinada forma y desarrollar conductas específicas al respecto.

De acuerdo con tal premisa, se diseñó un modelo teórico que propone que los sujetos poseen una serie de creencias sobre las enfermedades mentales, desarrolladas a partir de los elementos informativos recibidos por medio de diversos agentes de socialización (familia, escuela, medios de comunicación), y en determinados casos, a partir de experiencias personales concretas. Con base en tales creencias, se desarrollan actitudes e intenciones conductuales que, influenciadas por la necesidad experimentada y la disponibilidad de servicios de atención percibida, conducen a conductas específicas en relación con el apoyo a los enfermos mentales y la búsqueda oportuna de ayuda profesional.

Sobre esta base, el objetivo específico del trabajo que se presenta consistió en probar un modelo teórico diseñado para explicar las actitudes y conductas en relación con la enfermedad mental, a partir del conocimiento de las principales variables relacionadas con dicho constructo.

Las variables que integran el modelo y que fueron evaluadas se clasificaron de la siguiente manera:

A) Variables antecedentes

1) Creencias (acerca de las enfermedades mentales, sus causas, manifestaciones y posibilidades de tratamiento)

salud pública de méxico / vol.44, no.4, julio-agosto de 2002 
2) Fuentes de las creencias (información obtenida en la escuela, de la familia o a partir de los medios de comunicación)

3) Experiencias (directas con enfermos o enfermedades mentales)

4) Riesgo percibido (de padecer en lo personal, o en algún familiar cercano, una enfermedad mental)

5) Necesidad experimentada (de apoyo o asistencia especializada)

6) Disponibilidad percibida (de servicios de atención para trastornos mentales).

B) Variables intermedias

1) Actitudes (hacia las enfermedades y los enfermos mentales)

2) Intenciones conductuales (de reconocimiento y búsqueda de atención especializada).

C) Variable dependiente

1) Conducta reportada (de búsqueda de ayuda y aceptación de la enfermedad mental).

De acuerdo con este planteamiento, se diseñó el modelo que se esquematiza en la figura 1.

\section{Material y métodos}

Se efectuó una encuesta a una muestra de 800 sujetos de la población general de la Ciudad de México, es-

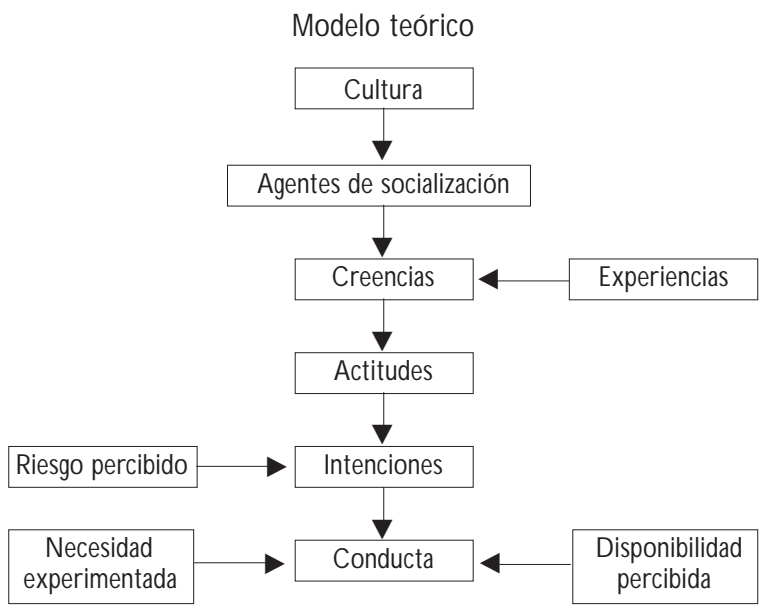

Figura 1. Modelo teórico básico para explicar la CONDUCTA EN RELACIÓN CON LA ENFERMEDAD MENTAL. MÉxıco, D.F., Méxıco, 1996 tratificada por nivel socioeconómico (medio y bajo), edad (cuatro grupos: 18-24, 25-34, 35-44, 45-60) y género (masculino y femenino). Las entrevistas se realizaron en el mes de mayo de 1996. Para la determinación y selección de la muestra, así como para la coordinación del trabajo de campo, se contó con la colaboración de la agencia de investigación mercadológica Moctezuma y Asociados. Se partió de la selección de 80 colonias, 40 clasificadas como de clase media y 40 de clase baja, de acuerdo con el mapa mercadológico elaborado por el Buró de Investigación de Mercados, S.A. de C.V. (BIMSA). Las 80 colonias se encuentran distribuidas en 12 delegaciones políticas del Distrito Federal y seis municipios del estado de México, ubicados en la zona conurbada de la Ciudad de México. En cada colonia se realizaron 10 entrevistas, seleccionando los hogares de manera intencional. En cada domicilio se entrevistó sólo un sujeto. En ningún caso se entrevistaron sujetos en domicilios contiguos. En el caso de unidades habitacionales se consideró un máximo de cinco edificios por unidad y un máximo de dos hogares por edificio. De esta manera se completó el total de las 800 entrevistas programadas.

Se construyó y validó un instrumento de medición diseñado específicamente para este estudio, integrado por 120 reactivos tipo Likert, con cinco opciones de respuesta, para medir las creencias, actitudes e intenciones conductuales. Las demás variables se evaluaron por medio de una sección adicional integrada por 30 reactivos. El instrumento fue aplicado en forma de entrevista estructurada, en el domicilio de los sujetos seleccionados, por aplicadores profesionales previamente entrenados.*

Los datos obtenidos fueron codificados y analizados estadísticamente por medio del paquete SPSS. En primer lugar, se evaluó la validez y confiabilidad de las escalas utilizadas, midiendo su consistencia interna por medio del Alfa de Cronbach, así como la validez del constructo mediante análisis factoriales. Posteriormente, se efectuaron pruebas $t$ de Student y anova para comparar a los grupos integrados por cada uno de los estratos de la muestra, a fin de detectar las posibles diferencias asociadas con características sociodemográficas y otras variables investigadas. Los resultados de estos análisis, han sido publicados en reportes previos. ${ }^{5,6}$

* El cuestionario utilizado puede solicitarse directamente a la autora. 


\section{Resultados}

En este artículo se presentan los resultados obtenidos en relación con la determinación de la capacidad predictiva del modelo teórico. Para tal fin, se realizaron análisis de regresión múltiple, estableciendo la contribución de las diferentes variables a la predicción de la variable subsecuente en la cadena causal, de acuerdo con el modelo propuesto.

Para cada modelo de regresión, se analizó la $\mathrm{R}$ múltiple (Rm), la R2, la R2 ajustada (R2aj) y el error estándar (EE) obtenidos, así como el valor de F y la significancia estadística respectiva. Se consideraron las variables que entraron al modelo de regresión, sus puntajes B y Beta, el error estándar, el valor de $t$ y su significancia estadística.

\section{Creencias}

En primer lugar, se efectuaron regresiones considerando como variable dependiente cada uno de los factores que integraron la escala de creencias sobre la enfermedad mental, y como independientes las experiencias tenidas y las tres posibles fuentes de las creencias, según hubieran sido transmitidas por cada uno de los agentes de socialización considerados: la familia, la escuela y los medios de comunicación.

Para el primer factor, correspondiente a las creencias sobre las características de las enfermedades mentales, se obtuvo un valor para la $\mathrm{Rm}=0.13$ y una $\mathrm{R} 2=$ 0.02 , lo que indica que las variables independientes consideradas explican en su conjunto sólo $2 \%$ del total de la varianza. La R2aj fue de 0.01 y el error estándar obtenido fue de 0.51 . Este modelo obtuvo una $F=3.39(p<0.009)$; la escuela y los medios de comunicación tuvieron poder predictivo en relación con el factor 1 de las creencias hacia la enfermedad mental.

El modelo correspondiente al segundo factor, sobre las causas de las enfermedades mentales, tuvo un valor para la $R m=0.04$ y una $R 2=0.001$, lo que indica que las variables independientes consideradas explican en su conjunto sólo $0.16 \%$ del total de la varianza. La R2aj fue de -0.003 y el error estándar obtenido fue de 0.45 . Este modelo obtuvo una $F=0.326$ (no significativa); ninguna variable tuvo poder predictivo en relación con este factor de las creencias hacia la enfermedad mental.

En cuanto al tercer factor, referente al tratamiento de la enfermedad mental, el modelo tuvo un valor para la $R m=0.13$ y una $R 2=0.02$, lo que indica que las variables independientes consideradas explican en su conjunto sólo $2 \%$ del total de la varianza. La R2aj fue de 0.013 y el error estándar obtenido fue de 0.70 . Este modelo obtuvo una $F=3.63$ ( $p<.0061)$; sólo las experiencias tuvieron poder predictivo en relación con el factor 3 de las creencias hacia la enfermedad mental. Estos datos se resumen en el cuadro I.

\section{Actitudes}

A continuación, se efectuaron análisis de regresión múltiple, considerando como variable dependiente cada uno de los factores de la escala de actitudes hacia la enfermedad mental, y como independientes los factores de creencias relacionados con el mismo.

En el primer caso, correspondiente al primer factor de las actitudes hacia la enfermedad mental, que agrupa reactivos relacionados con el temor, el modelo tuvo un valor para la $\mathrm{Rm}=0.14$ y una $\mathrm{R} 2=0.02$, lo que indica que las variables independientes consideradas explican en su conjunto $2 \%$ del total de la varianza. La R2aj fue de 0.015 y el error estándar obtenido fue de 0.80 . Este modelo obtuvo una $F=5.06(p<0.001)$,

\section{Cuadro I \\ Variables PRedictoras de las CREencias SOBRE LA ENFERMEDAD MENTAL. Ciudad de México, México, 1996}

Variable $B$ EE B Beta $t$ Sig.t

Factor 1: Características de la enfermedad mental

\begin{tabular}{lrrrrr} 
Escuela & -0.051 & 0.025 & -0.076 & -2.061 & 0.040 \\
\hline Medios de comunicación & 0.028 & 0.014 & 0.076 & 2.015 & 0.044 \\
\hline Familia & 0.042 & 0.022 & 0.070 & 1.894 & 0.059 \\
\hline Experiencias & 0.009 & 0.008 & 0.041 & 1.146 & 0.252 \\
\hline Constante & 3.796 & 0.044 & & 85.753 & 0.000
\end{tabular}

Factor 2: C ausas de la enfermedad mental

\begin{tabular}{lrrrrr} 
Escuela & 0.023 & 0.022 & 0.038 & 1.025 & 0.306 \\
\hline Experiencias & -0.003 & 0.007 & -0.013 & -0.351 & 0.726 \\
\hline Familia & -0.004 & 0.020 & -0.007 & -0.191 & 0.849 \\
\hline Medios de comunicación & 0.002 & 0.012 & 0.005 & 0.139 & 0.890 \\
\hline Constante & 3.041 & 0.039 & & 77.883 & 0.0000
\end{tabular}

Factor 3:Tratamiento de la enfermedad mental

\begin{tabular}{lrrrrr} 
Experiencias & 0.039 & 0.011 & 0.122 & 3.453 & 0.000 \\
\hline Familia & -0.054 & 0.031 & -0.065 & -1.750 & 0.080 \\
\hline Medios de comunicación & 0.012 & 0.019 & 0.024 & 0.650 & 0.516 \\
\hline Escuela & 0.021 & 0.034 & 0.022 & 0.602 & 0.547 \\
\hline Constante & 3.302 & 0.061 & & 54.100 & 0.000
\end{tabular}


siendo las creencias sobre las características de las enfermedades mentales la variable con mayor poder predictivo en relación con las actitudes de temor hacia las mismas.

Para el segundo factor de las actitudes hacia la enfermedad mental, correspondiente a las actitudes negativas, el modelo tuvo un valor para la $\mathrm{Rm}=0.38 \mathrm{y}$ una $R 2=0.15$, lo que indica que las variables independientes consideradas explican en su conjunto $15 \%$ del total de la varianza. La R2aj fue de 0.14 y el error estándar obtenido fue de 0.57 . Este modelo obtuvo una $F=45.28(p<0.0000)$, siendo también las creencias sobre las características de la enfermedad mental la variable con poder predictivo significativo en relación con las actitudes negativas.

En cuanto al tercer factor, que agrupa actitudes de tendencia positiva hacia la enfermedad mental, el modelo tuvo un valor para la $\mathrm{Rm}=0.36$ y una $\mathrm{R} 2=0.13$, lo que indica que las variables independientes consideradas explican en su conjunto $13 \%$ del total de la varianza. La R2aj fue de 0.12 y el error estándar obtenido fue de 0.68 . Este modelo obtuvo una $F=39.18$ $(p<0.001)$, siendo las creencias sobre los tratamientos para la enfermedad mental la variable con mayor poder predictivo en relación con las actitudes positivas, seguida de las creencias sobre las características de dichas enfermedades. Estos resultados se presentan en el cuadro II.

\section{Intenciones conductuales}

Posteriormente se realizaron análisis similares para cada uno de los factores de la escala de intenciones conductuales, considerando como variables independientes las actitudes y el riesgo percibido.

En el caso del primer factor, correspondiente a las intenciones positivas hacia la enfermedad mental, el modelo tuvo un valor para la $\mathrm{Rm}=0.28$ y una $\mathrm{R} 2=0.08$, lo que indica que las variables independientes explican en su conjunto $8 \%$ del total de la varianza. La R2aj fue de 0.07 y el error estándar obtenido fue de 0.635 . Este modelo obtuvo una $F=16.52(p<0.001)$, siendo los tres factores de las actitudes sobre la enfermedad mental y el riesgo, variables con poder predictivo en relación con las intenciones conductuales positivas.

En cuanto al segundo factor, correspondiente a las intenciones negativas hacia la enfermedad mental, el modelo tuvo un valor para la $\mathrm{Rm}=0.41$ y una $\mathrm{R} 2=0.17$, lo que indica que las variables independientes consideradas explican en su conjunto $17 \%$ del total de la varianza. La R2aj fue de 0.16 y el error estándar obtenido fue de 0.62 . Este modelo obtuvo una $F=40.57$ $(p<0.001)$, siendo las actitudes negativas y el temor hacia la enfermedad mental, en ese orden, las variables con poder predictivo en relación con las intenciones negativas al respecto (cuadro III).

\section{Cuadro II}

Variables predictoras de las actitudes hacia la enfermedad mental. Ciudad de México, México, 1996

Variable

Factor 1:Actitudes de temor

Creencias/C aracterísticas de la enfermedad mental

C reencias/Tratamiento de la enfermedad mental

Creencias/C ausas de la enfermedad mental

Constante
EE B

Beta

Sig. $t$

\begin{tabular}{lllll}
0.146 & 0.055 & -0.093 & -2.640 & 0.008 \\
\hline 0.077 & 0.040 & 0.067 & 1.910 & 0.056 \\
\hline 0.120 & 0.063 & -0.067 & -1.908 & 0.057 \\
\hline 3.570 & 0.325 & & 10.987 & 0.000
\end{tabular}

Factor 2:Actitudes negativas

\begin{tabular}{lccccc} 
Creencias/Tratamiento de la enfermedad mental & 0.457 & 0.039 & 0.380 & 11.575 & 0.000 \\
\hline Creencias/C aracterísticas de la enfermedad mental & -0.014 & 0.029 & -0.016 & -0.494 & 0.622 \\
\hline Creencias/C ausas de la enfermedad mental & -0.017 & 0.045 & -0.012 & -0.378 & 0.706 \\
\hline Constante & 2.139 & 0.232 & & 9.215 & 0.000
\end{tabular}

Factor 3:Actitudes positivas

C reencias/C aracterísticas de la enfermedad mental

Creencias/Tratamiento de la enfermedad mental

C reencias/C ausas de la enfermedad menta

Constante

0.35

0.35

0.152

$-0.064$

1.698

$\begin{array}{ll}0.034 & 0.34 \\ 0.047 & 0.10 \\ 0.053 & -0.04 \\ 0.274 & \end{array}$

\begin{tabular}{rrr}
0.348 & 10.479 & 0.000 \\
\hline 0.108 & 3.258 & 0.001 \\
-0.040 & -1.204 & 0.229 \\
\hline & 6.188 & 0.000
\end{tabular}




\section{Conducta}

Finalmente, se tomó como variable dependiente la conducta reportada y se incluyeron como independientes los dos factores de intenciones conductuales respecto de la enfermedad mental, la necesidad experimentada y la disponibilidad de servicios percibida; este modelo tuvo un valor para la $\mathrm{Rm}=0.79$ y una $\mathrm{R} 2=0.62$, lo que indica que las variables independientes consideradas explican en su conjunto $62 \%$ del total de la varianza. La R2aj fue de 0.62 y el error estándar obtenido fue de 1.27. Este modelo obtuvo una $F=324.03 \quad(p<0.001)$, siendo la necesidad experimentada la variable con mayor poder predictivo, seguida por la disponibilidad percibida de servicios de atención para trastornos mentales (cuadro III).

En la figura 2 se presentan los resultados obtenidos aplicados al esquema propuesto; como se aprecia, éstos se ajustan sólo parcialmente al modelo teórico. Esto es, se encontraron coeficientes de regresión importantes sólo entre los factores correspondientes a las creencias, las actitudes y las intenciones conductuales, corroborándose que las primeras, en efecto, pueden predecir las actitudes y éstas a su vez las intenciones conductuales. Sin embargo, las fuentes de las creencias no quedaron explicadas satisfactoria- mente a partir de las variables propuestas; las experiencias, el riesgo y la disponibilidad percibida no contribuyen de manera sensible a explicar el modelo, y la conducta resultó aparentemente poco influenciada por las intenciones, dependiendo de manera importante de la necesidad experimentada.

\section{Conclusiones}

Si bien los resultados de la investigación han sido satisfactorios en sus niveles descriptivos y correlacionales, reportados previamente, ${ }^{5,6}$ los análisis realizados para someter a prueba el modelo propuesto y comprobar su capacidad predictiva han permitido probar su adecuación y utilidad como base teórica sólo parcialmente. Es decir, se confirmó su capacidad predictiva solamente en la parte nuclear, correspondiente a la propuesta teórica de Fishbein y Ajzen, ${ }^{39-41}$ en cuanto a la cadena causal que conecta creencias, actitudes e intenciones conductuales; sin embargo, el interés fundamental por lograr una aproximación a la explicación de la conducta manifestada en relación con las enfermedades mentales, plantea aún serios interrogantes.

Aunque los resultados mostraron creencias, actitudes e intenciones conductuales positivas, en concordancia con la mayoría de los estudios recientes, éstas

\section{Cuadro III \\ VARIABLES PREDICTORAS DE LAS INTENCIONES Y LA CONDUCTA HACIA LA ENFERMEDAD MENTAL. Ciudad de México, Méxıco, 1996}

\begin{tabular}{lccccc} 
Variable & B & EE B & Beta & t & Sig.t \\
actor 1: Intenciones positivas & & & & & \\
Actitudes negativas hacia la enfermedad mental & 0.202 & 0.038 & 0.189 & 5.344 & 0.000 \\
\hline Actitudes positivas hacia la enfermedad mental & 0.118 & 0.032 & 0.129 & 3.660 & 0.000 \\
\hline Actitudes de temor hacia la enfermedad mental & -0.081 & 0.029 & -0.099 & -2.827 & 0.005 \\
\hline Riesgo percibido & 0.033 & 0.013 & 0.090 & 2.546 & 0.011 \\
\hline Constante & 2.860 & 0.198 & & 14.443 & 0.000
\end{tabular}

Factor 2: Intenciones negativas

\begin{tabular}{lccccc} 
Actitudes negativas hacia la enfermedad mental & 0.441 & 0.037 & 0.401 & 11.947 & 0.000 \\
\hline Actitudes de temor hacia la enfermedad mental & 0.056 & 0.028 & -0.066 & -1.994 & 0.047 \\
\hline Riesgo percibido & 0.011 & 0.013 & 0.030 & 0.909 & 0.364 \\
\hline Actitudes positivas hacia la enfermedad mental & 0.020 & 0.031 & 0.021 & 0.627 & 0.531 \\
\hline Constante & 2.315 & 0.193 & & 11.980 & 0.000
\end{tabular}

Conducta

\begin{tabular}{lccccc} 
N ecesidad & 3.396 & 0.096 & 0.779 & 35.404 & 0.000 \\
\hline Disponibilidad & 0.054 & 0.025 & 0.048 & 2.187 & 0.029 \\
\hline Intenciones negativas hacia la enfermedad mental & 0.106 & 0.068 & 0.035 & 1.560 & 0.119 \\
\hline Intenciones positivas hacia la enfermedad mental & 0.107 & 0.070 & 0.034 & 1.525 & 0.128 \\
\hline Constante & -1.208 & 0.388 & & -3.111 & 0.002
\end{tabular}


Modelo resultante

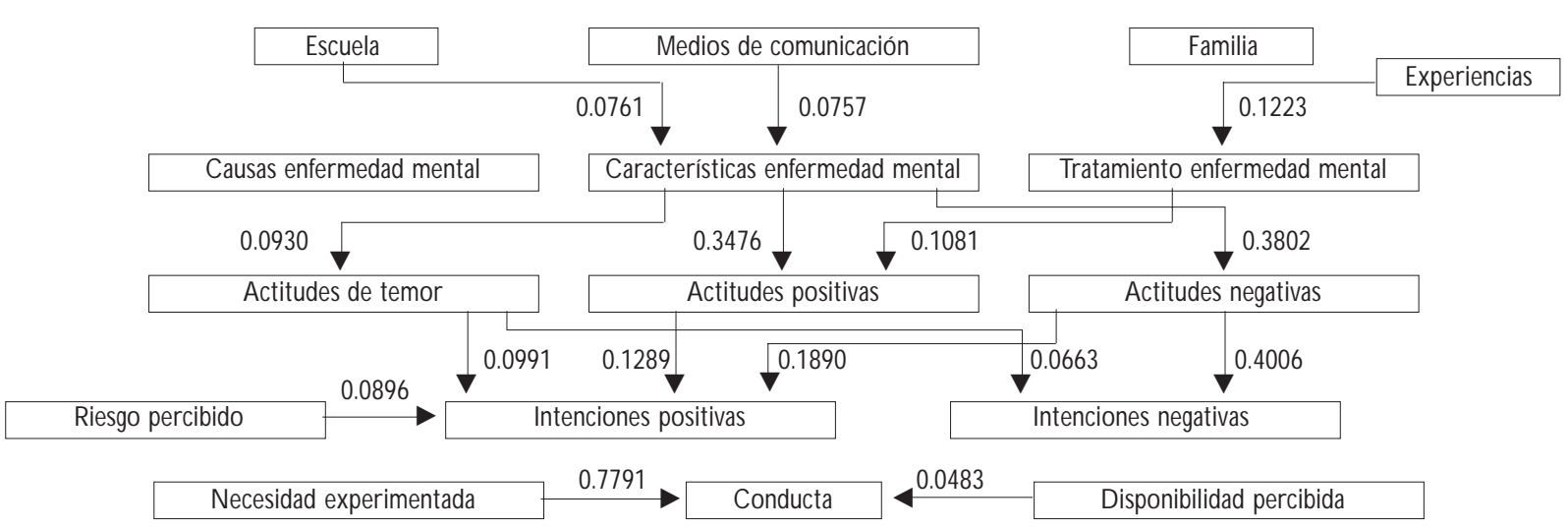

Figura 2. Modelo teórico ampliado para explicar la conducta en relación con la enfermedad mental. México, D.F., Méxıco, 1996

variables no explicaron en absoluto las conductas reportadas. Esto es, aunque la población parece tener bastante información acerca de las enfermedades mentales y afirma estar dispuesta a aceptar la cercanía de los enfermos mentales, en los hechos las conductas de búsqueda de atención, de reconocimiento de los síntomas y de aceptación de los enfermos en la familia o la comunidad mostraron depender, exclusivamente, de la magnitud de la necesidad experimentada (sólo cuando es extrema e imperativa) y en mucho menor grado, de la disponibilidad (de servicios) percibida.

Una posible explicación al hecho de que la conducta responda fundamentalmente a la necesidad experimentada por los sujetos y no parezca relacionarse con las creencias, actitudes e intenciones, podría considerar la posibilidad de que éstas correspondan a respuestas socialmente deseables, que podrían estar enmascarando sentimientos más profundos de tendencia negativa, lo cual explicaría que las opiniones y actitudes detectadas no siempre conducen a conductas activas de aceptación y búsqueda oportuna de atención.

En el ámbito de la salud mental pública, tanto la aceptación social de los enfermos como la disposición de éstos para buscar ayuda profesional son fundamentales; en México hasta la fecha, la atención de los trastornos mentales se basa primordialmente en los pacientes que acuden o son llevados a la consulta, lo que generalmente ocurre cuando los problemas ya tienen una larga historia y se han vuelto críticos; en la mayoría de los casos, el abordaje sería más conveniente, y el pronóstico más favorable, si una detección más tem- prana de los síntomas y una disposición más favorable para buscar ayuda llevara a mayor número de pacientes a la obtención oportuna de apoyo profesional. En este caso, como en muchos otros del ámbito de la salud, los programas preventivos se han basado en el conocimiento de las actitudes y en la premisa de que actitudes más positivas conducirán a las correspondientes conductas; sin embargo, en la práctica los resultados no siempre confirman la validez de tal premisa.

Parece ser que el cambio de actitudes que se ha observado en años recientes no es suficiente para modificar el comportamiento, por lo que es necesario poner un mayor énfasis en los programas de divulgación y educación acerca de las enfermedades y los enfermos mentales, y desplazar el estudio de las actitudes hacia la investigación sobre la utilización de servicios y el desarrollo y evaluación de programas de sensibilización e intervención.

La importancia de la educación en salud mental destaca como medio para propiciar el reconocimiento temprano de los síntomas de las diversas enfermedades mentales y para inducir a una búsqueda más oportuna de atención, ya que la utilización de los servicios psiquiátricos disponibles no depende sólo de su existencia, sino de la apropiada educación masiva para la salud mental. Los resultados obtenidos constituyen un punto de partida para proseguir en el desarrollo de investigaciones más específicas y profundas en cada una de las áreas incluidas, especialmente en aquellas que no pudieron ser adecuadamente explicadas por el modelo, esto es, investigaciones que intenten profun- 
dizar en la discrepancia aparente entre actitudes y comportamiento, considerando tanto el estudio de otras posibles variables intervinientes, como la utilización de metodologías complementarias, fundamentalmente las de tipo cualitativo, que podrían aportar nueva luz a tan complejo problema.

\section{Referencias}

1.Ayuso JL, Saiz J.A comparative study of the psychiatric nurses' attitudes towards mental patients. Int J Soc Psychiatry 1978;24(1):47-52.

2. Brockington IF, Hall P, Levings J, Murphy C. The community's tolerance of the mentally ill. Br J Psychiatry 1993;162 (93):99.

3. Reda S. Public perception of discharged psychiatric patients: A community survey. Int J Soc Psychiatry 1996;42(3):220-229.

4.W olff G, Pathare S, C raigT, Leff J. Community attitudes to mental illness. Br J Psychiatry 1996;168:183-190.

5. García-Sílberman S. Attitudes toward mental illness and psychiatry: Preliminary results. Salud Mental 1998;21(4):40-50.

6. García-Sílberman S. Creencias acerca de la enfermedad mental. En: Asociación Mexicana de Psicología Social, ed. La psicología social en México. México, D.F.:AMEPSO , 2000: 338-344.

7.A guirre D. El estigma de la enfermedad mental (tesis) México, D.F.: Universidad N acional Autónoma de México, 1983.

8. Casco M. Percepción y actitud ante los problemas de salud mental entre jóvenes de educación media superior. Salud Mental 1990;13(2):18-23. 9. Casco M, N atera G. Percepción de un grupo de profesionistas hacia la imagen que la comunidad tiene de la enfermedad mental: comparación entre ambas poblaciones. Salud Mental 1986;9(2): 70-77.

10. Chávez MI. La solicitud de ayuda profesional: su relación con la atribución de enfermedad mental y con el nivel de tolerancia social a las alteraciones conductuales (tesis). México, D.F.:U niversidad Iberoamericana, 1982. 11. De la Serna MT. Análisis del concepto de enfermedad mental en una comunidad suburbana (tesis), México, D.F.: Universidad Iberoamericana, 1982.

12. González SR. La actitud hacia la enfermedad mental (tesis). México, D.F.: Universidad N acional Autónoma de México, 1986.

13. Gutiérrez E. Actitud de la Comunidad hacia la enfermedad mental (tesis). México, D.F.: U niversidad N acional Autónoma de México, 1983.

14. $N$ atera $G, C$ asco M. Percepción de la enfermedad mental a través de historietas. Bol 0 ficina Sanit Panam 1985;98(4): 327-338.

15. N atera G, Casco M. Actitudes hacia la enfermedad mental en población general y en un grupo de profesionales de la salud. En:Anales del Instituto Mexicano de Psiquiatría. México, D.F.: IMP, 1991: 176-182.

16. Parra F. Social tolerance of the mentally ill in the Mexican American community. Int J Soc Psychiatry 1985;31: 37-45.

17. Parra F. Perceptions of mental illness in Mexico:A descriptive study in the city of Chihuahua. Int J Soc Psychiatry 1987;33(4):270-276.

18. Parra FYiu-Cheong A. The changing perceptions of mental illness in a Mexican-A merican community. Int J Soc Psychiatry 1983;29: 95-99.

19. Bhugra D.Attitudes toward mental illness:A review of the literature. Acta Psychiatr Scand 1989;80:1-6.
20. Brockman J, D 'A rcy C. Correlates of attitudinal social distance toward the mentally ill:A review and re-survey. Soc Psychiatry 1978;13:69-77.

21. C asco M, N atera G, Herrejón ME. La actitud hacia la enfermedad mental, una revisión de la bibliografía. Salud Mental 1987;10(2):41-50.

22. Rabkin J. Public attitudes toward mental illness:A review of the literature. Schizophr Bull 1974;10(Fall):9-33.

23. Ruiz M, Serrano V, Sánchez E. Historia de las actitudes hacia la enfermedad mental. Actas Luso-Españolas de Neurologia y Psiquiatria 1988;16(4):285-293.

24. Angermeyer $M C$, Matschinger $H$. The effect of personal experience with mental illness on the attitude towards individuals suffering from mental disorders. Social Psychiatry Psychiatr Epidemiol 1996;31:321-326.

25. Angermeyer $\mathrm{MC}$, Matschinger $\mathrm{H}$. Social distance towards the mentally ill: Results of representative surveys in the Federal Republic of Germany. Psychol Med 1997;27:131-141.

26. Bissland JH, Munger R. Implications of changing attitudes toward mental illness. J Soc Psychol 1985;125(4):515-517.

27. Chou KL, Mak KY, C hung PK, Ho K.Attitudes towards mental patients in Hong-Kong. Int J Soc Psychiatry 1996;42(3):213-219.

28. Eker D. University students' attitudes toward mental patients in a developing country. Soc Psychiatry Psychiatr Epidemiol 1988;23:264-266.

29. Eker D. Attitudes toward mental illness: Recognition, desired social distance, expected burden and negative influence on mental health among Turkish freshmen. Soc Psychiatry Psychiatr Epidemiol 1989;24:146-150.

30. Madianos MG, Economou M, Hatjiandreou, Papageorgiou A, Rogakou E. Changes in public attitudes towards mental illness in the Athens area (1979/1980-1994).Acta Psychiatr Scand 1999;99:73-78.

31. Matschinger $\mathrm{H}$, Angermeyer MC. Lay beliefs about the causes of mental disorders: A new methodological approach. Soc Psychiatry Psychiatr Epidemiol 1996;31:309-315.

32. Penayo U, Jacobsson L, Caldera T, Bermann G. Community attitudes and awareness of mental disorders.Acta Psychiatr Scand 1988;78: 561-566.

33. Read J, Law A.The relationship of causal beliefs and contact with users of mental health services to attitudes to the 'mentally ill'. Int J Soc Psychiatry 1999:45(3):216-229.

34. Shurka E. Attitudes of Israeli Arabs towards the mentally ill. Int J Soc Psychiatry 1983;29:101-110.

35.Taylor SM, D ear MJ. Scaling community attitudes toward the mentally ill. Schizophr Bull 1981;7(2):225-239.

36. Trute B, Teftt B, Segall A. Social rejection of the mentally ill: A replication study of public attitude. Soc Psychiatry Psychiatr Epidemiol 1989;24: 69-76.

37. W olff G, Pathare S, C raigT, Leff J. C ommunity know ledge of mental illness and reaction to mentally ill people. Br J Psychiatry 1996;168: 191-198.

38. Blalock HM. Construcción de teorías en ciencias sociales: de las formulaciones verbales a las matemáticas. México, D.F.:Trillas, 1986.

39. Fishbein M, Ajzen I. Belief, attitude, intention and behavior: An introduction to theory and research. N ueva York (N Y):Addison-W esley, 1975. 40.Ajzen I.Attitude structure and behavior. En: Pratkanis AR, Breckler S), $G$ reenwald $A G$, ed. Attitude structure and function. Hillsdal $(O \mathrm{H}): 0$ hio State University/Lawrence Erlbaum Assoc. Publishers, 1989.

41. Ajzen I, Fishbein M. Understanding attitudes and predicting social behavior. N ueva Jersey (N J): Prentice-Hall, 1980. 